\title{
DISCOVERY OF A SMALL CENTRAL DISK OF CO AND H I IN THE MERGER REMNANT NGC 34
}

\author{
Ximena Fernández ${ }^{1}$, A. O. Petric ${ }^{2,3}$, François Schweizer ${ }^{4}$, And J. H. VAn Gorkom ${ }^{1}$ \\ ${ }^{1}$ Department of Astronomy, Columbia University, New York, NY 10027, USA; ximena@astro.columbia.edu \\ ${ }^{2}$ Department of Astronomy, California Institute of Technology, Pasadena, CA 91125, USA \\ ${ }^{3}$ Gemini Science Fellow, Gemini Observatory, 670 N. A’ohoku Pl., Hilo, HI 96720, USA \\ ${ }^{4}$ Carnegie Observatories, 813 Santa Barbara Street, Pasadena, CA 91101, USA \\ Received 2013 October 4; accepted 2014 January 8; published 2014 February 27
}

\begin{abstract}
We present $\mathrm{CO}(1-0)$ and $\mathrm{H}$ I $(21 \mathrm{~cm})$ observations of the central region of the wet merger remnant NGC 34 . The Combined Array for Research in Millimeter-wave Astronomy observations detect a regularly rotating disk in CO with a diameter of $2.1 \mathrm{kpc}$ and a total molecular hydrogen mass of $(2.1 \pm 0.2) \times 10^{9} M_{\odot}$. The rotation curve of this gas disk rises steeply, reaching maximum velocities at $1^{\prime \prime}(410 \mathrm{pc})$ from the center. Interestingly, H I observations performed with the Karl G. Jansky Very Large Array show that the absorption against the central continuum has the exact same velocity range as the $\mathrm{CO}$ in emission. This strongly suggests that the absorbing $\mathrm{H}$ I also lies within $1^{\prime \prime}$ from the center, is mixed in, and corotates with the molecular gas. A comparison of $\mathrm{H}$ I absorption profiles taken at different resolutions $\left(5^{\prime \prime}-45^{\prime \prime}\right)$ shows that the spectra at lower resolutions are less deep at the systemic velocity. This provides evidence for $\mathrm{H}_{\mathrm{I}}$ emission in the larger beams, covering the region from $1 \mathrm{kpc}$ to $9 \mathrm{kpc}$ from the center. The central rapidly rotating disk was likely formed either during the merger or from fall-back material. Lastly, the radio continuum flux of the central source at mm wavelengths $(5.4 \pm 1.8 \mathrm{mJy})$ is significantly higher than expected from an extrapolation of the synchrotron spectrum, indicating the contribution of thermal free-free emission from the central starburst.
\end{abstract}

Key words: galaxies: evolution - galaxies: individual (NGC 34, NGC 17, Mrk 938) - galaxies: interactions galaxies: Seyfert - galaxies: starburst - radio continuum: galaxies

Online-only material: color figures

\section{INTRODUCTION}

Gas-rich mergers are key to understanding the hierarchical growth and evolution of galaxies (White \& Rees 1978). Much progress has been made to understand the overall role of mergers in the formation of early-type galaxies, and it is now accepted that many are a product of gas-rich mergers (e.g., Schweizer 1998). There are still some uncertainties on how exactly the transformation takes place, especially how a gas-rich system ultimately rids itself of gas. These details are what possibly dictate whether the merger remnant becomes an elliptical or whether a new disk forms, as seen in some observations and simulations of gas-rich mergers (Schiminovich et al. 2013; Barnes 2002). The possible presence of a starburst or active galactic nucleus (AGN) further complicates the picture. While gas can fuel both starbursts and AGNs, these phenomena may cause outflows and remove the gas from the inner parts. It is thus important to study the gas in the central regions of mergers in its molecular and atomic phases to help predict the ultimate fate of the system.

NGC 34 (= NGC $17=$ Mrk 938) is an ideal candidate for studying these processes, since it is gas-rich and hosts both a starburst and an AGN. Throughout the paper, we adopt a distance of $85.2 \mathrm{Mpc}$, derived for $H_{0}=70 \mathrm{~km} \mathrm{~s}^{-1} \mathrm{Mpc}^{-1}$ by Schweizer \& Seitzer (2007, hereafter SS07). The infrared luminosity of this object is $\log \left(L_{\mathrm{IR}} / L_{\odot}\right)=11.61$ (Chini et al. 1992), placing it in mid-range of the classically defined Luminous Infrared Galaxies (LIRGs; $11.0<\log L_{\mathrm{IR}} / L_{\odot}<12.0$; Soifer et al. 1987). In general, high IR luminosities are due to dust grains being heated by a combination of a starburst and AGN, with higher values indicating a greater AGN contribution (Sanders \& Mirabel 1996). The IR luminosity of NGC 34 suggests that the starburst is the dominant phenomenon in the center, with a minor AGN component (Gonçalves et al. 1999; Prouton et al. 2004). This has been confirmed by a detailed study of NGC 34's IR spectrum performed by Esquej et al. (2012), where the AGN bolometric contribution to the total IR luminosity is estimated to be $2_{-1}^{+2} \%$. In addition, the IR images show that the starburst takes place in the inner $0.5-2 \mathrm{kpc}$ of the merger remnant. These authors find, however, that the presence of an AGN is needed to explain the hard X-ray luminosity.

An optical study by SS07 showed that NGC 34 is the likely product of an unequal-mass merger, with a rich system of young globular clusters. Deep optical images show two long tidal tails and structures such as ripples, jets, and shells, all typical of merger remnants (Schweizer 1998). In addition to this, the surface brightness profiles in $B, V$, and $I$ indicate the presence of a blue exponential stellar disk with a scale length of $a=3.3 \mathrm{kpc}$, possibly formed from gas falling back into the merger remnant. The optical spectrum features a blueshifted NaI D doublet in absorption, indicative of a mean gas outflow velocity of $-620 \pm$ $60 \mathrm{~km} \mathrm{~s}^{-1}$ and a maximum velocity of $-1050 \pm 30 \mathrm{~km} \mathrm{~s}^{-1}$.

Earlier, we performed a first imaging study of the H I and radio continuum to better understand the fate of cold gas during the merger (Fernández et al. 2010, hereafter Radio I). We detected $7.2 \times 10^{9} M_{\odot}$ of $\mathrm{HI}$ gas, which is mostly distributed along the tails, and saw evidence for gas settling onto the blue exponential disk found by SS07. The most puzzling part of our study was the detection of a strong $\mathrm{H}$ I absorption feature of $\sim 500 \mathrm{~km} \mathrm{~s}^{-1}$ width at the systemic velocity, covering both blueshifted and redshifted velocities and seen against the strong unresolved central continuum source of $62.4 \mathrm{mJy}$. We presented two possible scenarios to explain the puzzling absorption: either we were probing an H I disk in absorption against an extended 
continuum source so that we saw both the blueshifted and redshifted parts in absorption, or we were seeing gas associated with the tidal tails in projection against the continuum.

In this follow-up radio study, we present new $\mathrm{CO}$ imaging and $\mathrm{H}$ I absorption observations obtained at higher angular resolution and with a wider velocity coverage. We seek to determine which of the above two scenarios explains the puzzling absorption better by comparing the distribution and kinematics of the $\mathrm{CO}$ to the $\mathrm{HI}_{\mathrm{I}}$ absorption data. In addition, the new continuum observations at $\mathrm{mm}$ wavelengths allow us to probe the spectral index at high frequencies. Lastly, both sets of new observations have a much wider velocity coverage, enabling us to search for an atomic or molecular counterpart to the outflow seen optically.

The structure of the paper is as follows. We summarize the observations and data reduction in Section 2, present our results in Section 3, discuss what we have learned from these observations in Section 4, and put forth our conclusions in Section 5 .

\section{OBSERVATIONS AND DATA REDUCTION}

\subsection{CARMA Observations}

NGC 34 was observed in the CO $J=1-0$ transition at 115.2712 GHz with the Combined Array for Research in Millimeter-wave Astronomy (CARMA) in 2011 May. The observations were performed in two configurations: in the $\mathrm{D}$ array for $4.1 \mathrm{hr}$ on source and in the $\mathrm{C}$ array for $5.8 \mathrm{hr}$. The spacings for these two arrays are 11-150 $\mathrm{m}$ and 30-350 m, respectively. The primary flux calibrator was Uranus, and 3C454.3 was used as the passband calibrator. Both sets of observations used a $1 \mathrm{GHz}$ bandwidth centered at $113.11 \mathrm{GHz}$, corresponding to the central frequency of the single-dish observations. This translates to a heliocentric velocity of $5731 \mathrm{~km} \mathrm{~s}^{-1}$, using the optical definition. This setup results in a velocity coverage of $2980 \mathrm{~km} \mathrm{~s}^{-1}$, starting at $4238 \mathrm{~km} \mathrm{~s}^{-1}$ and ending at $7218 \mathrm{~km} \mathrm{~s}^{-1}$, with 150 channels with a velocity resolution of $20 \mathrm{~km} \mathrm{~s}^{-1}$.

The data were reduced with the software package MIRIAD using standard calibration procedures for each configuration (Sault et al. 1995). For each band, we flagged the first and last two channels due to poor sensitivity at the edge of the band. The phase stability was inspected and poor data were flagged for each track. The $\mathrm{C}$ and $\mathrm{D}$ array calibrated visibilities were then combined and the continuum was estimated from 696 (unsmoothed) signal free channels. The deconvolution task uses a Steer CLEAN algorithm (Steer et al. 1984), and we selected natural weighting (robustness parameter set to 2) to maximize the sensitivity to broad, faint structures. The final combined data cube has a synthesized beam of $2^{\prime \prime} .48 \times 2$ '. $14(\sim 1 \mathrm{kpc})$ at FWHM and a noise of $8.4 \mathrm{mJy}$ beam $^{-1}$.

\subsection{VLA Observations}

NGC 34 was observed with the Karl G. Jansky Very Large Array $(\mathrm{VLA})^{5}$ in 2011 April in the $\mathrm{B}$ array configuration (spacings of $0.21-11.1 \mathrm{~km}$ ) during two runs for a total observing time of $7 \mathrm{hr}$. The observations used a $32 \mathrm{MHz}$ bandwidth centered at the heliocentric velocity of $5870 \mathrm{~km} \mathrm{~s}^{-1}$, with a total of 256 channels with a resolution of $27 \mathrm{~km} \mathrm{~s}^{-1}$. This results

\footnotetext{
5 The National Radio Astronomy Observatory is a facility of the National Science Foundation operated under cooperative agreement by Associated Universities, Inc.
}

Table 1

Observation Parameters

\begin{tabular}{|c|c|c|}
\hline & $\mathrm{CO}(1-0)^{\mathrm{a}}$ & $\mathrm{HI}^{\mathrm{b}}$ \\
\hline Flux calibrator & Uranus & $3 \mathrm{C} 48$ \\
\hline Phase calibrator & $3 \mathrm{C} 454.3$ & $\mathrm{~J} 2357-1125$ \\
\hline Number of channels & 150 & 256 \\
\hline Bandwidth (MHz) & 1000 & 32 \\
\hline Synthesized beam & $2^{\prime \prime} .48 \times 2^{\prime \prime} .14$ & $8^{\prime \prime} .31 \times 4^{\prime \prime} .46$ \\
\hline Field of view & $1-1^{\prime} .7$ & $32^{\prime}$ \\
\hline $\begin{array}{l}\text { Velocity coverage } \\
\left(\mathrm{km} \mathrm{s}^{-1}\right)\end{array}$ & 3000 & 7000 \\
\hline $\begin{array}{l}\text { Velocity resolution } \\
\left(\mathrm{km} \mathrm{s}^{-1}\right)\end{array}$ & 20 & 27 \\
\hline $\begin{array}{l}\text { Noise } \\
\qquad(\mathrm{mJy} \text { beam } \\
\end{array}$ & 8.4 & 0.21 \\
\hline $\begin{array}{l}\text { Column density sensitivity } \\
\qquad\left(\mathrm{cm}^{-2} \operatorname{chan}^{-1}\right)\end{array}$ & $1.1 \times 10^{20}\left(\mathrm{H}_{2}\right)$ & $1.7 \times 10^{20}$ \\
\hline
\end{tabular}

Notes.

a Observed in 2011 May.

b Observed in 2011 April.

c In emission.

in a velocity coverage of $7000 \mathrm{~km} \mathrm{~s}^{-1}$, from $2426 \mathrm{~km} \mathrm{~s}^{-1}$ to $9421 \mathrm{~km} \mathrm{~s}^{-1}$.

Each run was reduced with AIPS using standard calibration procedures. The data were combined in the $u v$ plane, and then several iterations of self-calibration were performed to correct for amplitude and phase errors. We first made a continuum image by averaging 80 line-free channels, using a robustness parameter of 1 and setting CLEAN boxes around the point source. This image served as the initial input model for the first run of phase self-calibration. After each run, we made a new input model by imaging the continuum, applying the new solutions for subsequent self-calibration runs. After three runs of phase self-calibration and one of amplitude, we achieved a dynamic range of about 760:1 and an rms sensitivity of 0.07 mJy beam ${ }^{-1}$. We then applied the solutions to the whole data set, and subtracted the continuum in the $u v$ plane by making a linear fit through the line-free channels. The H I cube was made using a robustness parameter of 1 and cleaning the channels containing $\mathrm{H}_{\mathrm{I}}$ absorption. The resulting images have an rms noise of $0.2 \mathrm{mJy} \mathrm{beam}^{-1}$ and a synthesized beam of $8 . .31 \times 4^{\prime \prime} .46$ $(\sim 3.5 \times 2 \mathrm{kpc})$.

Table 1 summarizes all parameters used for the CARMA and VLA observations.

\section{RESULTS}

\subsection{A Central Disk of $\mathrm{CO}$}

The CO observations reveal a disk of molecular gas in the central regions of the remnant. Figure 1 is composed of various panels showing the gas distribution and kinematics. This $\mathrm{CO}$ disk lies at the center of NGC 34 and is much smaller than the optical disk (Figure 1(a)). It shows a regular rotation pattern as seen in Figures 1(d) and (e). There are hints of extended emission toward the northwest, northeast, and south (Figure 1(b)). We estimate a diameter of $2.1 \mathrm{kpc}$ by measuring the extent of the emission enclosed by the second lowest density contour, which corresponds to $4.31 \mathrm{Jy} \mathrm{km} \mathrm{s}^{-1}$ beam $^{-1}$.

We calculate the CO luminosity with the following equation (Solomon et al. 1997):

$$
L_{\mathrm{CO}}=3.25 \times 10^{7} S d v v_{\mathrm{obs}}^{-2} D_{L}^{2}(1+z)^{-3},
$$




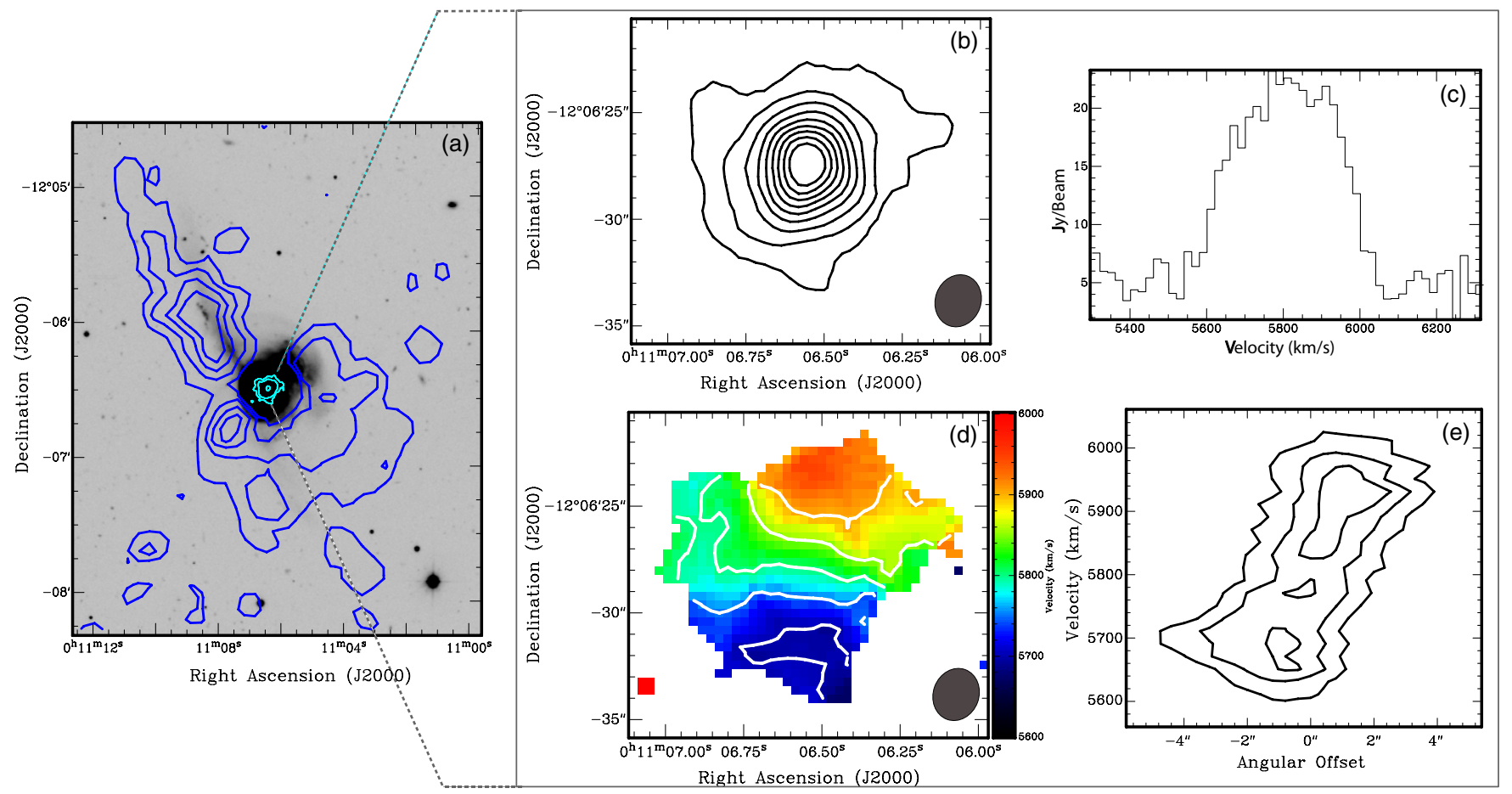

Figure 1. Maps of the distribution and kinematics of the CO disk found in NGC 34. (a) CO emission as compared to the optical and H I emission; the cyan contours show the CO emission, and the H I contours (in blue; from Radio I) are drawn in levels of $(8,28,48,68,108) \times 10^{19} \mathrm{~cm}^{-2}$ overlaid on an optical image from SS07. (b) CO distribution map (moment 0 ) contours drawn starting at $5 \%$ of the peak, in intervals of $10 \%$. (c) The spectrum of the CO emission was obtained by setting a box around the emission showing how much $\mathrm{CO}$ there is in a given velocity bin. (d) Velocity map (moment 1) overlaid with isovelocity contours drawn in intervals of $50 \mathrm{~km} \mathrm{~s}^{-1}$. (e) Position-velocity diagram along the major axis of the optical disk (P.A. -9 ${ }^{\circ}$; SS07). Note that the full range of velocities is not shown in these maps; we limit them to show a better contrast or to zoom-in in a region of interest.

(A color version of this figure is available in the online journal.)

where $S d v$ is the integrated CO flux in units of $\mathrm{Jy} \mathrm{km} \mathrm{s}^{-1}, v_{\mathrm{obs}}$ is the observed frequency in $\mathrm{GHz}$, and $D_{L}$ is the luminosity distance in Mpc. We compute the $\mathrm{CO}$ flux using channel maps and get a value of $151.9 \pm 11.3 \mathrm{Jy} \mathrm{km} \mathrm{s}^{-1}$, which results in a CO luminosity of $(2.6 \pm 0.2) \times 10^{9} \mathrm{~K} \mathrm{~km} \mathrm{~s}^{-1} \mathrm{pc}^{-2}$. We multiply this value by $\alpha_{\mathrm{CO}}$ to get a molecular hydrogen mass in solar masses $\left(M_{\odot}\right)$. Here we use $\alpha=0.8$, which is the standard conversion factor for starbursting systems (Downes \& Solomon 1998), and get an $\mathrm{H}_{2}$ mass of $2.1 \pm 0.2 \times 10^{9} \mathrm{M}_{\odot}$.

Single-dish CO(1-0) data (Kandalyan 2003; Chini et al. 1992; Kruegel et al. 1990) yield a flux of $170 \pm 12 \mathrm{Jy} \mathrm{km} \mathrm{s}^{-1}$, which is $12 \%$ higher than what we detect. This is a marginal difference, but - if significant-it might indicate that our observations miss an extended component of the $\mathrm{CO}$ disk. This component is hinted at by the weak extensions seen in Figure 1(b), suggesting that observing with shorter spacings could have detected this more extended gas.

As seen in the spectrum of Figure 1(c), the $\mathrm{CO}$ emission spans close to $500 \mathrm{~km} \mathrm{~s}^{-1}$. The intensity-weighted velocity field (Figure 1(d)) and the position-velocity (PV) diagram (Figure 1(e)) along the major axis of the optical disk (P.A. $-9^{\circ}$; SS07) show that the kinematics are consistent with rotation, with the north side of the disk receding, and the south side approaching. The extended emission features mentioned above have velocities consistent with the gas in the main body of the disk, suggesting they are real and possibly part of a fainter component not detected in our observations. The PV diagram demonstrates that the rotation curve rises very steeply, reaching peak velocities within $1^{\prime \prime}$ from the center and showing a slight decrease toward the edges.

\subsection{High Resolution H I Absorption}

Our new VLA B array observations probe the previously known H I absorption feature (Radio I) with a wider velocity coverage and at higher resolution. Figure 2 consists of three panels showing these observations, including a comparison to the absorption profiles presented in Radio I, and to the CO observations in emission presented in Section 3.1. The top panel represents the $\mathrm{HI}$ absorption feature seen against the peak of the continuum emission and showing the full velocity coverage of 2426-9421 km s${ }^{-1}$, with no hint of absorption seen at the outflow velocity $\left(\sim 5000 \mathrm{~km} \mathrm{~s}^{-1}\right)$ detected by SS07 (see the next section).

The second panel compares these higher resolution $(2 \mathrm{kpc})$ data to the previous $\mathrm{H}$ I observations in the $\mathrm{DnC}(18 \mathrm{kpc})$ and $\mathrm{CnB}(6 \mathrm{kpc})$ configurations. Note that the depth of absorption is similar in the three configurations at the extreme velocities around $5600 \mathrm{~km} \mathrm{~s}^{-1}$ and $6000 \mathrm{~km} \mathrm{~s}^{-1}$, but is very different near the systemic velocity of $5870 \mathrm{~km} \mathrm{~s}^{-1}$. A deep feature is seen in the $\mathrm{B}$ array at this velocity, which is shallower in $\mathrm{C}$ and nearly absent at some velocities in D. We can interpret this as follows: Since the continuum source is unresolved in the B array, we see a combination of absorption against the continuum source plus emission further out within the central beam in the C and D arrays. We can estimate how much emission there is on different scales by looking at the difference in the absorption profiles. We do this by summing the difference between the two profiles at different resolutions for each velocity bin to get a flux. As shown in Radio I, there is $7.3 \times 10^{8} M_{\odot}$ of $\mathrm{H}$ I in emission between the inner 6 and $18 \mathrm{kpc}$ of the remnant. Now we can determine how 

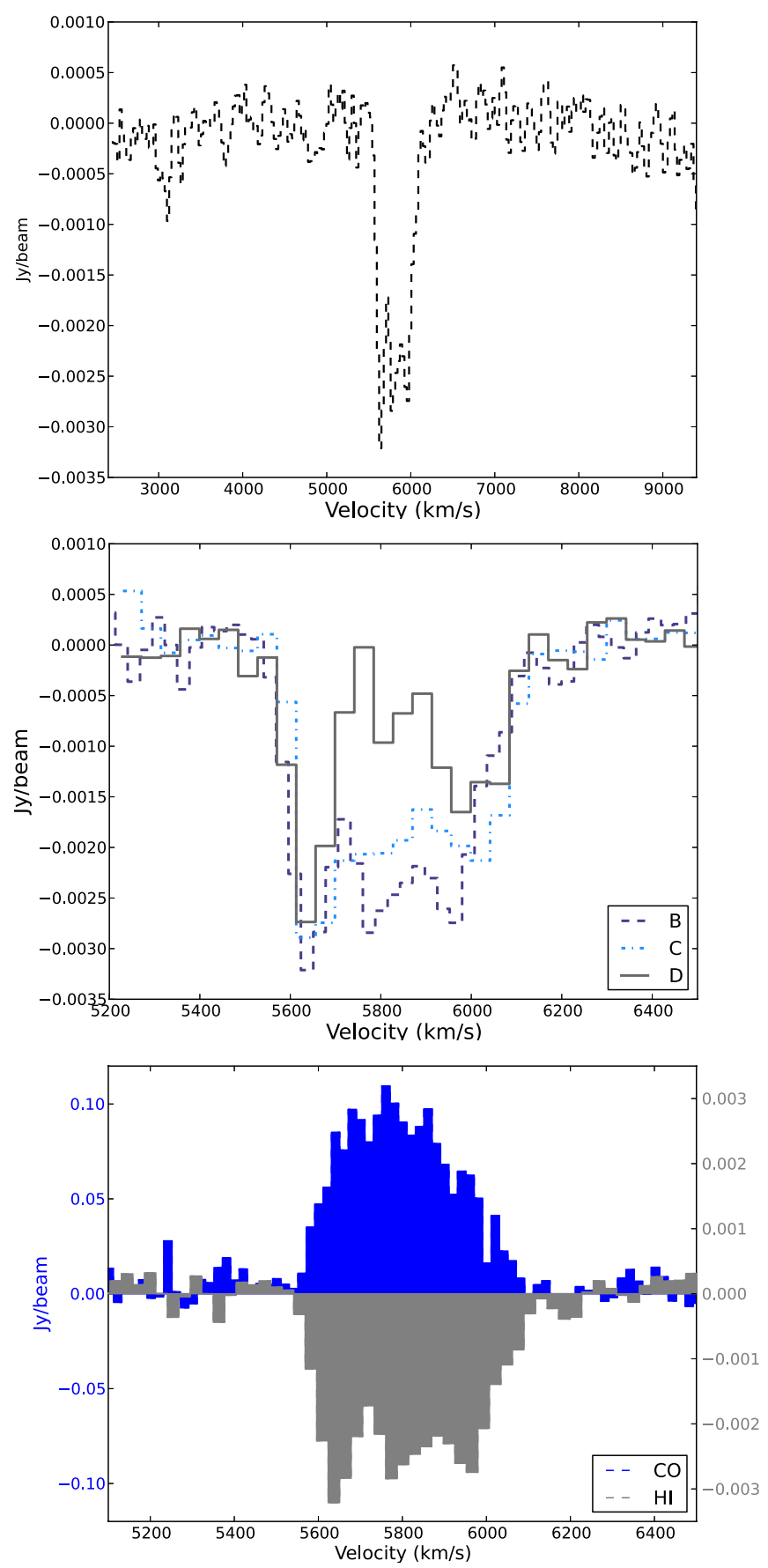

Figure 2. H I absorption at higher resolution and wider velocity coverage. Top: B array observations showing the $\mathrm{H}$ I absorption at both blueshifted and redshifted velocities. Middle: comparison of the absorption profile observed at three different resolutions (DnC: $18 \mathrm{kpc}, \mathrm{CnB}: 6 \mathrm{kpc}$, and B: $2 \mathrm{kpc}$ ). Bottom: comparison of the $\mathrm{H}$ I absorption profile with the $\mathrm{CO}$ emission spectrum. The $y$-axes are different for the two, but are shown in the same plot to compare the velocities.

(A color version of this figure is available in the online journal.)

much $\mathrm{H}_{\mathrm{I}}$ in emission there is between the inner 2 and $6 \mathrm{kpc}$ by comparing the $\mathrm{CnB}$ and the $\mathrm{B}$ array data. We calculate an $\mathrm{H}_{\mathrm{I}}$ mass of $2.5 \times 10^{8} M_{\odot}$ in the velocity interval $5700-6000 \mathrm{~km} \mathrm{~s}^{-1}$. Since the two data sets have different velocity resolutions, we created velocity bins of $50 \mathrm{~km} \mathrm{~s}^{-1}$ and then averaged the values that were in a given bin.

The bottom panel compares the range of velocities seen in the $\mathrm{H}$ I absorption and the $\mathrm{CO}$ emission. The plot shows that the velocity ranges for the emission and the absorption are almost identical. The range covered for the $\mathrm{HI}$ absorption profile is $466 \pm 27 \mathrm{~km} \mathrm{~s}^{-1}$ and for the CO profile is $460 \pm 20 \mathrm{~km} \mathrm{~s}^{-1}$, as measured at the profile base. This remarkable agreement in velocity range makes it plausible that the $\mathrm{H}$ I and $\mathrm{CO}$ emissions arise from the same central gas disk, imaged in CO at 2". $48 \times 2$ ". 14 resolution (Figures 1 (b) and (d)). In addition, it implies that the $\mathrm{H} \mathrm{I}$ is intermixed with the molecular gas and that it also reaches maximum velocities within $\sim 1^{\prime \prime}(0.4 \mathrm{kpc})$ from the center.

\subsection{Molecular and Atomic Gas Outflow}

We can use the current observations to search for a counterpart in $\mathrm{HI}_{\mathrm{I}}$ and/or $\mathrm{CO}(1-0)$ to the outflow detected by SS07 in the NaI D doublet. We do not detect the outflow in either line, but can place upper limits. We calculate the noise near the central regions of NGC 34 and around $\sim 5000 \mathrm{~km} \mathrm{~s}^{-1}$, consistent with the velocities of the optical outflow. We use the following equation to get a mass outflow rate upper limit (Heckman et al. 2000; Rupke et al. 2002) for both H I absorption and emission:

$$
\begin{aligned}
\dot{M}(H)= & 21\left(\frac{\Omega}{4 \pi}\right) C_{f}\left(\frac{r_{*}}{1 \mathrm{kpc}}\right)\left(\frac{N(H)}{10^{21} \mathrm{~cm}^{-2}}\right) \\
& \times\left(\frac{\Delta v}{200 \mathrm{~km} \mathrm{~s}^{-1}}\right) M_{\odot} \mathrm{yr}^{-1}
\end{aligned}
$$

where $\Omega$ is the solid angle subtended by the outflow, $C_{f}$ is the line-of-sight covering fraction, $r_{*}$ is the size of the region from where the outflow is produced, $N(H)$ is the column density, and $\Delta v$ is the velocity of the outflow. For the first three quantities, we assume $\Omega=4 \pi, C_{f}=0.5$, and $r_{*}=1 \mathrm{kpc}$, which are values consistent with outflows seen in LIRGs (Rupke et al. 2002). For the absorption, we calculate the H I column density assuming $5 \sigma$ and get $4.2 \times 10^{20} \mathrm{~cm}^{-2}$ (assuming $T_{s}=100 \mathrm{~K}$ ). Lastly, we take $\Delta v=620 \mathrm{~km} \mathrm{~s}^{-1}$, which is the mean velocity of the outflow detected by SS07. The resulting upper limit for the mass outflow rate is $14 M_{\odot} \mathrm{yr}^{-1}$ in absorption. Using the same values, but now calculating $\mathrm{N}(\mathrm{HI})$ in emission, the upper limit is about $130 M_{\odot} \mathrm{yr}^{-1}$. This large number is a consequence of the poor surface brightness sensitivity in the B array.

From the $\mathrm{CO}$ emission, we can calculate an upper limit for the outflow rate by dividing the molecular mass by the dynamical timescale (e.g., Alatalo et al. 2011). We compute an upper limit for the molecular-gas mass using $5 \sigma$ and the velocity of the optical outflow, resulting in $6.2 \times 10^{7} M_{\odot}$. We can estimate the dynamical time by assuming a size of $1 \mathrm{kpc}$ for the wind emitting region and the optical velocity, which results in a timescale of only 1.6 Myr. These two values yield an upper limit for the CO mass outflow rate of about $40 M_{\odot} \mathrm{yr}^{-1}$.

\subsection{Central Continuum}

This study includes new continuum images of the central components of the merger remnant. The VLA B array observations show a slightly resolved point source with a flux of $60.6 \pm 2.0 \mathrm{mJy}$, consistent with the measurement presented in Radio I.

The CARMA observations reveal a faint central continuum source at millimeter wavelengths with a flux of $5.4 \pm 1.8 \mathrm{mJy}$. This measurement allows us to probe the spectral index at high frequencies. At low frequencies $(1-10 \mathrm{GHz})$, the spectrum for star-forming galaxies is usually dominated by synchrotron radiation, with typical values of $\alpha=-0.8$. At higher frequencies (10-200 GHz), free-free emission starts to dominate and the 


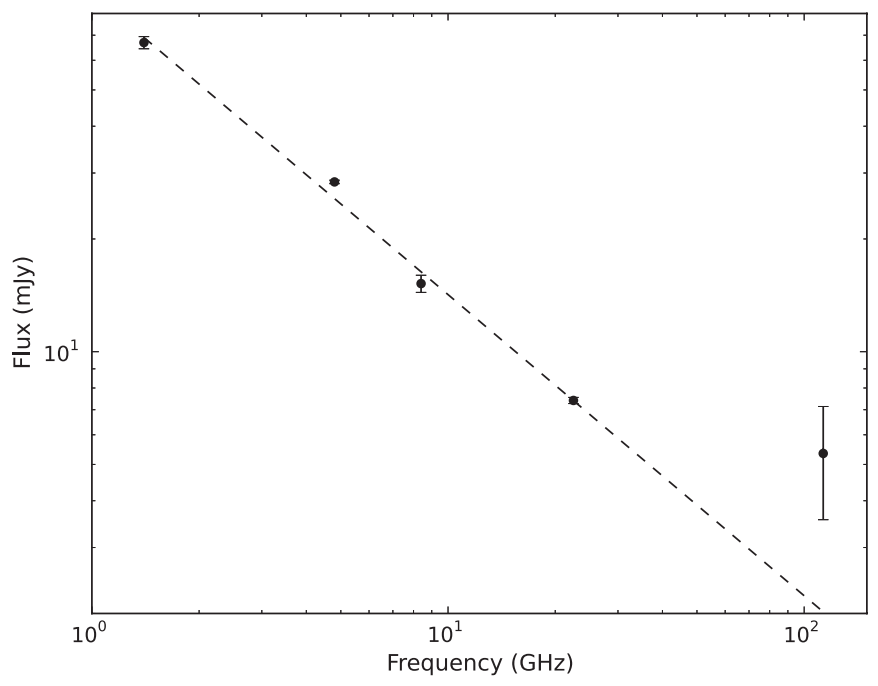

Figure 3. Flux measurements at different frequencies, with the first four measurements taken from Clemens et al. (2008) and the measurement at $113 \mathrm{GHz}$ showing the new observations presented here. The line marks the least-squares fit to the first four measurements (slope of -0.8 ), but is extended to demonstrate that the spectral index changes at higher frequencies.

spectral index is $\alpha=-0.1$. Dust emission takes over at frequencies above $200 \mathrm{MHz}$ with $\alpha=1.5$ (Condon 1992).

Figure 3 shows the radio spectrum for NGC 34 between 1.4 GHz and $113.11 \mathrm{GHz}$. The first four values in the range of $1.4-22.5 \mathrm{GHz}$ were reported by Clemens et al. (2008), and the one at $113.11 \mathrm{GHz}$ is the measurement presented here. We fit a line through the first four measurements and extend it to $113.11 \mathrm{GHz}$ to better show that the spectrum flattens at higher frequencies. The fit of the line yields a slope of -0.8 , indicating that the emission at these frequencies is due to synchrotron radiation. The spectral index between $22.5 \mathrm{GHz}$ and $113.11 \mathrm{GHz}$ is $\alpha=-0.20 \pm 0.07$. The flattening of the spectrum at higher frequencies shows that synchrotron emission is no longer dominant, and free-free emission from $\mathrm{H}$ II regions takes over.

\section{DISCUSSION}

\subsection{The Gaseous Disk in the Inner Regions of the Remnant}

Our CO observations confirm the presence of a disk in the inner regions of the remnant, which was one of the two scenarios suggested in Radio I to explain the H I absorption observations. The match in velocity for both sets of observations (Figure 2) strongly suggests that the central $\mathrm{HI}$ absorption is due to the presence of a rotating disk. As shown in the PV diagram of Figure 1(e), the maximum velocities are reached within only $1^{\prime \prime}(0.4 \mathrm{kpc})$ from the center. The close match in velocity between the molecular gas and $\mathrm{H}$ I absorption argues that the absorbing gas also reaches maximum velocities within $0.4 \mathrm{kpc}$ from the center. This is consistent with higher resolution continuum images by Condon et al. (1991) that resolve the central source and show it to be a dominant central component with an extension to the south, with a total size of $2.5(1 \mathrm{kpc})$. This extended continuum makes it possible to trace out the $\mathrm{H}$ I absorption to its maximum velocity at $1^{\prime \prime}$ from the center.

The question now is whether any of the continuum emission could be due to the AGN. Several recent studies have calculated the AGN contribution to be small (1\%-10\%) in NGC 34 (e.g., Vega et al. 2008; Esquej et al. 2012; Murphy 2013). In addition to this, VLBI observations report a single-baseline detection (Lonsdale et al. 1993), which suggests that this merger remnant does not host a strong point source due to the AGN, and its continuum is likely dominated by the starburst.

\subsection{The Fate of Gas in the Merger}

The optical study by SS07 and the radio observations presented here and in Radio I have shown different components of the new disk formed after the merger. SS07 find a blue stellar disk, with spiral structure extending out to a radius of $\sim 3.3 \mathrm{kpc}$, that seems to have formed $\sim 400 \mathrm{Myr}$ ago and is embedded in a red spheroid. In Radio I, we found that the $\mathrm{H}$ I from the northern tidal tail is falling back and continuing to feed the outer regions of this optical disk, favoring the idea of inside-out growth. On smaller scales, we have shown in this study that there is a central disk of $\mathrm{CO}$ and $\mathrm{H}$ I of about $2.1 \mathrm{kpc}$ in diameter. In addition, we find evidence for more extended $\mathrm{H}$ i emission further out as shown by the comparison of the absorption profiles at different resolutions.

Observations and simulations of mergers suggest that about $50 \%$ of the gas gets quickly moved to the central regions, losing its angular momentum due to cloud-cloud collisions, while the other $50 \%$ gets moved to large distances from the center, but remains bound and will eventually fall back (Hibbard \& Mihos 1995; Hibbard \& van Gorkom 1996; Barnes 2002). In NGC 34 we see evidence for both processes, and currently there are comparable amounts of cold gas in the tidal tails and the center. We know the blue stellar disk formed $\sim 400 \mathrm{Myr}$ ago from gas settling toward the center during the merger. The central gaseous disk presented here could have formed during the merger or more recently from fall-back material. The major axes of the gaseous disk and the blue stellar disk have almost identical position angles, indicating that the two disks either have the same formation mechanism or are dynamically linked. The current star formation rate (SFR) in the inner $2 \mathrm{kpc}$ calculated by Esquej et al. (2012) from the $24 \mu \mathrm{m}$ luminosity is $42 M_{\odot} \mathrm{yr}^{-1}$, which implies a consumption timescale of $\sim 50 \mathrm{Myr}$ for the molecular hydrogen presented here. This indicates that the gas in the central disk must be continuously replenished to sustain the current SFR.

Lastly, both sets of observations have enough velocity coverage to permit searching for a possible radio counterpart to the outflow seen optically in NaI D absorption by SS07. However, we do not find any evidence for such a counterpart in the atomicor molecular-gas phase. We are able to place approximate upper limits on the $\mathrm{H}$ I mass outflow rate in both emission and absorption, and for the $\mathrm{CO}$ in emission.

\section{SUMMARY AND CONCLUSIONS}

We have presented new high resolution $\mathrm{H}$ I and $\mathrm{CO}$ observations to study the central regions of the merger remnant NGC 34. Our main findings are as follows.

1. We detect a rotating $\mathrm{CO}$ disk of $2.1 \mathrm{kpc}$ in diameter with a molecular-hydrogen mass of $(2.1 \pm 0.2) \times 10^{9} \mathrm{M}_{\odot}$.

2. The velocity width of the $\mathrm{CO}(1-0)$ emitting gas matches the $\mathrm{HI}$ absorption width, indicating that the broad $\mathrm{HI}$ absorption is due to the central gas disk and is co-spatial with the $\mathrm{CO}$.

3. The new $\mathrm{B}$ array observations of the $\mathrm{H}$ I absorption feature allow us to calculate that there is $2.5 \times 10^{8} M_{\odot}$ of $\mathrm{HI}$ in the annular region of $1-3 \mathrm{kpc}$ from the center.

4. We do not detect a molecular- or atomic-gas outflow, but place upper limits on the mass outflow rates for $\mathrm{H}_{\mathrm{I}}$ in emission and absorption, and for $\mathrm{CO}$ in emission. 
5. We have obtained new continuum images of the central source at $1.4 \mathrm{GHz}$ and $113 \mathrm{GHz}$. We find that the spectrum flattens at the highest frequencies to a spectral index of $\alpha=-0.20 \pm 0.07$. This indicates that the radio continuum at those frequencies is due to free-free emission from the central starburst.

Support for CARMA construction was derived from the Gordon and Betty Moore Foundation, the Kenneth T. and Eileen L. Norris Foundation, the James S. McDonnell Foundation, the Associates of the California Institute of Technology, the University of Chicago, the states of California, Illinois, and Maryland, and the National Science Foundation. Ongoing CARMA development and operations are supported by the National Science Foundation under a cooperative agreement, and by the CARMA partner universities. This work was partially supported by NSF grant No. 1009476 to Columbia University.

\section{REFERENCES}

Alatalo, K., Blitz, L., Young, L. M., et al. 2011, ApJ, 735, 88

Barnes, J. E. 2002, MNRAS, 333, 481

Chini, R., Kruegel, E., \& Steppe, H. 1992, A\&A, 255, 87

Clemens, M. S., Vega, O., Bressan, A., et al. 2008, A\&A, 477, 95

Condon, J. J. 1992, ARA\&A, 30, 575

Condon, J. J., Huang, Z.-P., Yin, Q. F., \& Thuan, T. X. 1991, ApJ, 378, 65
Downes, D., \& Solomon, P. M. 1998, ApJ, 507, 615

Esquej, P., Alonso-Herrero, A., Pérez-Garca, A. M., et al. 2012, MNRAS, 423,185

Fernández, X., van Gorkom, J. H., Schweizer, F., \& Barnes, J. E. 2010, AJ, 140,1965

Gonçalves, A. C., Véron-Cetty, M.-P., \& Véron, P. 1999, A\&AS, 135, 437

Heckman, T. M., Lehnert, M. D., Strickland, D. K., \& Armus, L. 2000, ApJS, 129,493

Hibbard, J. E., \& Mihos, J. C. 1995, AJ, 110, 140

Hibbard, J. E., \& van Gorkom, J. H. 1996, AJ, 111, 655

Kandalyan, R. A. 2003, A\&A, 398, 493

Kruegel, E., Chini, R., \& Steppe, H. 1990, A\&A, 229, 17

Lonsdale, C. J., Smith, H. J., \& Lonsdale, C. J. 1993, ApJL, 405, L9

Murphy, E. J. 2013, ApJ, 777, 58

Prouton, O. R., Bressan, A., Clemens, M., et al. 2004, A\&A, 421, 115

Rupke, D. S., Veilleux, S., \& Sanders, D. B. 2002, ApJ, 570, 588

Sanders, D. B., \& Mirabel, I. F. 1996, ARA\&A, 34, 749

Sault, R. J., Teuben, P. J., \& Wright, M. C. H. 1995, in ASP Conf. Ser. 77, Astronomical Data Analysis Software and Systems IV, ed. R. A. Shaw, H. E. Payne, \& J. J. E. Hayes (San Francisco, CA: ASP), 433

Schiminovich, D., van Gorkom, J. H., \& van der Hulst, J. M. 2013, AJ, 145,34

Schweizer, F. 1998, in Galaxies: Interactions and Induced Star Formation, ed. D. Friedli, L. Martinet, \& D. Pfenniger (Berlin: Springer), 105

Schweizer, F., \& Seitzer, P. 2007, AJ, 133, 2132

Soifer, B. T., Sanders, D. B., Madore, B. F., et al. 1987, ApJ, 320, 238

Solomon, P. M., Downes, D., Radford, S. J. E., \& Barrett, J. W. 1997, ApJ, 478,144

Steer, D. G., Dewdney, P. E., \& Ito, M. R. 1984, A\&A, 137, 159

Vega, O., Clemens, M. S., Bressan, A., et al. 2008, A\&A, 484, 631

White, S. D. M., \& Rees, M. J. 1978, MNRAS, 183, 341 